\title{
A NOTE ON ORDERED PLANES
}

\author{
MARGARET A. GAMON
}

Department of Mathematics
Carroll College

Helena, Montana 59625-0099

and

D.C. KENT

Department of Mathematics

Washington State University

Pullman, Washington 99164-3113

(Received December 4, 1990 and in revised form May 15, 1991)

ABSTRACT. Necessary and sufficient conditions are given for the equivalence of the Nachbin and Wallman-ordered compactification of an ordered plane.

KEY WORDS AND PHRASES. Ordered topological space, ordered plane, $c$-space, $T_{4}$-ordered space, ordered compactification.

1980 MATHEMATICS SUBJECT CLASSIFICATION CODE : 54F05, 54D35

\section{INTRODUCTION.}

Our notation and terminology will agree with that of [4]. If $A$ is a non-empty subset of a poset $(X, \leq)$, we denote by $i(A)$ (respectively, $d(A)$ ) the increasing (respectively, decreasing) hull of $A$, defined by $i(A)=\{x \in X: a \leq x$ for some $a \in A\}$. A set $A$ is said to be increasing (respectively, decreasing) if $A=i(A)$ (respectively, $A=d(A)$ ); $A$ is convex if $A=i(A) \cap d(A)$.

We define an ordered topological space (or, for brevity, space) to be a triple $(X, \leq, T)$, where $(X, \leq)$ is a poset and $T$ is a convex topology (i.e., a topology with a subbase consisting of increasing and decreasing open sets). We will usually write $X$ rather $\operatorname{than}(X, \leq, \tau)$ when no ambiguity will result.

A space $X$ is $T_{1}$-ordered if $i(x)$ and $d(x)$ are closed for all $x \in X ; X$ is $T_{2}$-ordered if the order relation is closed in $X \times X$. If $X$ is normally ordered (in the sense of Nachbin, [5]) and $T_{2}$-ordered, then $X$ is said to be $T_{4}$-ordered. A space which is $T_{2}$-ordered and totally ordered is called a totally ordered space. We use the term real ordered space to describe a totally ordered space where underlying poset is the set of real numbers with their usual order (but not necessarily the usual topology). A product of two real ordered spaces is called an ordered plane.

Starting with a subset $A$ of a space $X$, let $I(A)$ be the closed, increasing hull of $A$ (i.e., the smallest closed, increasing set containing $A$ ); the closed, decreasing hull $D(A)$ is defined dually. $A$ is defined to be a $c$-set if $A=I(A) \cap D(A)$. A space $X$ is called a $c$-space (see [4]) if, for every $c$-set $A, i(A)$ and $d(A)$ are both closed sets. 
Each completely-regular-ordered space $X$ (as defined in [5]) allows $T_{2}$-ordered compactifications, the largest of which is the Nachbin (or Stone-Čech-ordered) compactification (see [1], [5]). In 1976, Choe and Park introduced the Wallman-ordered compactification of an arbitrary $T_{1}$-ordered space $X$, and it was shown in 1985 (see [3]) that $w_{0} X$ and $\beta_{0} X$ are equivalent if and only if $X$ is a $T_{4}$ ordered $c$-space. In this note, we show that an ordered plane is always $T_{4}$ ordered, and is a $c$-space if and only if it has one of four topologies. This gives necessary and sufficient conditions for $\beta_{0} P$ and $w_{0} P$ to coincide for an ordered plane $P$.

\section{ORDERED PLANES.}

In a real ordered space $X=(R, \tau)$, every point has a neighborhood base of convex sets. Then for any $x \in X$, basic neighborhoods are of exactly one of the following four types: $(x-\varepsilon, x+$ $\varepsilon),\{x, x+\varepsilon),(x-\varepsilon, x],\{x\}$. Of course, different points in $X$ may have different types of basic neighborhoods. Four well-known topologies on $R$ are designated as follows: (1) $\tau_{u}$ is the usual topology on $R$ with basic neighborhoods $(x-\varepsilon, x+\varepsilon)$ at every point $x \in R$; (2) $\tau_{d}$ is the discrete topology; (3) $T_{e}$ is the left-Sorgenfrey topology, with basic neighborhoods $(x, x+\varepsilon)$ at every point $x$; (4) $T_{r}$ is the right-Sorgenfrey topology, with basic neighborhoods $(x-\varepsilon, x]$ at every point $x$.

THEOREM 1. Let $P=X \times Y$ be an ordered plane. $P$ is a $c$-space iff $P$ has one of the following topologies: (1) $\tau_{u} \times \tau_{u},(2) \tau_{d} \times \tau_{d},(3) \tau_{e} \times \tau_{r},(4) \tau_{r} \times \tau_{e}$.

PROOF. Assume that $P$ has one of the four specified topologies. In Theorem 3.1, [4], it is proved that $P$, equipped with $\tau_{u} \times \tau_{u}$, is a $c$-space. It is obvious that any poset with the discrete topology is a $c$-space. Next, assume that $P$ has the topology $T_{l} \times T_{r}$. Suppose $A$ is closed and convex in $P$; we will show by contradiction that $i(A)$ is also closed.

If $i(A)$ is not closed, then there is $\left(x_{0}, y_{0}\right) \in \operatorname{cli}(A)$ (the closure of $\left.i(A)\right)$ such that $\left(x_{0}, y_{0}\right) \in i(A)$. Let $\left(x_{n}, y_{n}\right)$ be a sequence in $i(A)$ converging to $\left(x_{0}, y_{0}\right)$. Since $\left(x_{0}, y_{0}\right) \notin i(A), A \cap d\left(x_{0}, y_{0}\right)=\emptyset$. By assumption, all basic neighborhoods of $\left(x_{0}, y_{0}\right)$ are subsets of $S=\left\{(x, y): x \geq x_{0}, y \leq y_{0}\right\}$, and

we assume without loss of generality that $\left(x_{n}, y_{n}\right) \in S$, for all $n$. Indeed, since $\left(x_{0}, y_{0}\right) \notin i(A)$, we can assume that $\left(x_{n}, y_{n}\right) \in S_{0}=\left\{(x, y): x>x_{0}, y \leq y_{0}\right\}$ for all $n$.

Let $\left(a_{n}, b_{n}\right)$ be a sequence in $A$ such that $\left(a_{n}, b_{n}\right) \leq\left(x_{n}, y_{n}\right)$, for all $n$; it follows that $\left(a_{n}, b_{n}\right) \in S_{0}$ for all $n$. Since $x_{0}<a_{n} \leq x_{n}$ for all $n,\left(a_{n}\right) T_{r}$-converges to $x_{0}$. Furthermore $\left(b_{n}\right)$ has either an increasing or a decreasing subsequence; we consider both cases.

CASE 1. $\left(b_{n}\right)$ has an increasing subsequence $\left(b_{n_{k}}\right)$. Since $\left(b_{n_{k}}\right)$ is bounded above by $y_{0}$ and increasing, it must $\tau_{e}$-converge to some $b_{0}$. Then $\left(a_{n_{k}}, b_{n_{k}}\right)$ converges to $\left(x_{0}, b_{0}\right)$, and the latter point is in $A$ (since $A$ is closed). But $b_{0} \leq y_{0}$ implies $\left(x_{0}, y_{0}\right) \in i(A)$, a contradiction.

CASE 2. $\left(b_{n}\right)$ has a decreasing subsequence $\left(b_{n_{k}}\right)$. Let $\left(a_{n_{k_{j}}}\right)$ be a decreasing subsequence of $\left(a_{n_{k}}\right)$; if $a_{j}^{\prime}=a_{n_{k_{j}}}$ and $b_{j}^{\prime}=b_{n_{k_{j}}}$, then $\left(a_{j}^{\prime}, b_{j}^{\prime}\right)$ is a decreasing subsequence of $\left(a_{n}, b_{n}\right)$. For arbitrary $j$, we have $\left(a_{j}^{\prime}, b_{j}^{\prime}\right) \leq\left(a_{j}^{\prime}, b_{1}^{\prime}\right) \leq\left(a_{1}^{\prime}, b_{1}^{\prime}\right)$, and since $A$ is convex, $\left(a_{j}^{\prime}, b_{1}^{\prime}\right) \in A$ for all $j$. Thus the sequence $\left(a_{j}^{\prime}, b_{1}^{\prime}\right)$ converges to $\left(x_{0}, b_{1}^{\prime}\right)$, and $\left(x_{0}, b_{1}^{\prime}\right) \in A$ since $A$ is closed. Also, $b_{1}^{\prime} \leq y_{n_{k 1}} \leq y_{0}$; thus $\left(x_{0}, y_{0}\right) \in i(A)$, a contradiction.

We conclude that every point in cli(a) is also in $i(A)$, so $i(A)$ is closed. A dual argument shows that $d(A)$ is closed. Similar arguments apply if $P$ has the topology $\tau_{r} \times \tau_{l}$. Thus the proof is complete in one direction.

To prove the converse, observe that if $P$ is not equipped with one of the four specified topologies, then some point $\left(x_{0}, y_{0}\right)$ in $P$ has basic neighborhoods of one of the following twelve types: (1) $\left(x_{0}-\varepsilon, x_{0}+\varepsilon\right) \times\left\{y_{0}\right\} ;(2)\left[x_{0}, x_{0}+\varepsilon\right) \times\left\{y_{0}\right\} ;(3)\left[x_{0}, x_{0}+\varepsilon\right) \times\left[y_{0}, y_{0}+\varepsilon\right) ;(4)\left(x_{0}-\varepsilon, x_{0}+\varepsilon\right) \times\left[y_{0}, y_{0}+\varepsilon\right) ;(5)$ $\left\{x_{0}\right\} \times\left(y_{0}-\varepsilon, y_{0}\right] ;(6)\left\{x_{0}\right\} \times\left(y_{0}-\varepsilon, y_{0}+\varepsilon\right) ;(7)\left(x_{0}-\varepsilon, x_{0}\right] \times\left(y_{0}-\varepsilon, y_{0}\right) ;(8)\left(x_{0}-\varepsilon, x_{0}\right] \times\left(y_{0}-\varepsilon, y_{0}+\varepsilon\right) ;(9)$ $\left(x_{0}-\varepsilon, x_{0}\right] \times\left\{y_{0}\right\} ;(10)\left(x_{0}-\varepsilon, x_{0}+\varepsilon\right) \times\left(y_{0}-\varepsilon, y_{0}\right] ;(11)\left\{x_{0}\right\} \times\left[y_{0}, y_{0}+\varepsilon\right) ;(12)\left[x_{0}, x_{0}+\varepsilon\right) \times\left(y_{0}-\varepsilon, y_{0}+\varepsilon\right)$.

First assume there is a point $\left(x_{0}, y_{0}\right)$ with basic neighborhoods of one of the types (1), (2), (3), or (4). Let $A=\left\{(x, y): x=-y, x>x_{0}\right\}$. One may verify that for any of these four neighborhood 
types, $d(A)=D(A), I(A)=i(A) \cup\left\{\left(x_{0}, y\right): y \geq y_{0}\right\}$, and $A=I(A) \cap D(A)$. Thus $A$ is a $c$-set, and since $i(A)$ is not closed, $P$ is not a $c$-space.

Next assume there is a point $\left(x_{0}, y_{0}\right)$ with basic neighborhoods of one of the types (5), (6), (7), or (8). Let $A$ be defined as in the preceding paragraph. One can verify that $i(A)=I(A), D(A)=$ $d(A) \cup\left\{\left(x, y_{0}\right): x \leq x_{0}\right\}$, and $A=I(A) \cap D(A)$. Thus $A$ is a $c$-set and $A$ is not closed, so $P$ is not a $c$-space.

Finally, suppose there is a point $\left(x_{0}, y_{0}\right)$ with basic neighborhoods of one of types (9), (10), (11), or (12). Let $B=\left\{(x, y): x=-y\right.$ and $\left.y>y_{0}\right\}$. One can verify that $B$ is a $c$-set. If the basic neighborhoods at $\left(x_{0}, y_{0}\right)$ are of type (9) or (10), then $I(B)=i(B)$ and $D(B) \neq d(B)$. If the basic neighborhoods at $\left(x_{0}, y_{0}\right)$ are of type (11) or (12), then $D(B)=d(B)$ and $I(B) \neq i(B)$.

Thus for any topology on $P$ other than the four specified, $P$ fails to be a $c$-space, and the proof is complete. I

THEOREM 2. Every ordered plane is $T_{4}$-ordered.

PROOF. Let $P=X \times Y$ be an ordered plane. Let $A$ be a closed, increasing subset of $X \times Y$ and let $B$ be a closed, decreasing subset of $X \times Y$ such that $A \cap B=\emptyset$. The method of proof is to construct for each $x \in A$ and $y \in B$, an open, increasing neighborhood $N_{s_{.}}(x)$ of $x$ and an open, decreasing neighborhood $M_{c,}(y)$ of $y$ such that $N_{c s}(x) \cap B=\emptyset$ and $M_{c y}(y) \cap A=\emptyset$. We thess show that $N_{c_{s}}(x) \cap M_{c_{y}}(y)=\emptyset$, for all $x \in A$ and $y \in B$. Thus if $U=\cup_{x \in A} N_{\frac{c_{z}}{2}}(x)$ and $V=\cup_{y \in B} M_{\frac{c_{y}}{3}}(y)$, then $U$ is open, increasing, $V$ is open, decreasing, $A \subseteq U, B \subseteq V$, and $U \cap V=\emptyset$.

Let $x=\left(x_{1}, x_{2}\right) \in A$; the set $N_{c}(x)$ is defined for four different cases, where $\varepsilon>0$ is arbitrary.

CASE $1:\left[x_{1}, \infty\right)$ is open in $X,\left[x_{2}, \infty\right)$ is open in $Y$.

Let $N_{c}(x)=\left[x_{1}, \infty\right) \times\left[x_{2}, \infty\right)$. (Note that in this case, $N_{c}(x)$ is the same set for all $\varepsilon>0$.

CASE 2: $\left[x_{1}, \infty\right)$ open in $X,\left(x_{2}, \infty\right)$ not open in $Y$.

Let $N_{c}(x)=\left[x_{1}, \infty\right) \times\left(x_{2}-\varepsilon, \infty\right)$.

CASE 3: $\left[x_{1}, \infty\right)$ not open in $X,\left[x_{2}, \infty\right)$ open in $Y$.

Let $N_{c}(x)=\left(x_{1}-\varepsilon, \infty\right) \times\left[x_{2}, \infty\right)$.

CASE 4: $\left[x_{1}, \infty\right)$ not open in $X,\left[x_{2}, \infty\right)$ not open in $Y$.

Let $N_{\varepsilon}(x)=\left(x_{1}-\varepsilon, \infty\right) \times\left(x_{2}-\varepsilon, \infty\right)$.

CLAIM 1: For each $x \in A$, there is $\varepsilon_{\mathrm{z}}$ such that $N_{\mathrm{c}} \cap B=\emptyset$.

PROOF. In Case 1, $N_{\varepsilon} \subseteq A$, for all $\varepsilon$, so $\varepsilon_{z}$ may be chosen arbitrarily. In Case 2 , since $B$ is closed, there is some $\varepsilon_{x}$ such that $\left(\left\{x_{1}\right\} \times\left(x_{2}-\varepsilon_{x}, x_{2}\right]\right) \cap B=\emptyset$. Let this $\varepsilon_{x}$ (or any smaller one) be chosen. In Case 3, there likewise exists $\varepsilon_{x}$ such that $\left(\left(x_{1}-\varepsilon_{x}, x_{1}\right)\right.$; times $\left.\left\{x_{2}\right\}\right) \cap B=\emptyset$, again let this $\varepsilon_{x}$ be chosen. In Case 4, there exists $\varepsilon_{z}$ such that $\left(\left(x_{1}-\varepsilon_{x}, x_{1}\right] \times\left(x_{2}-\varepsilon_{x}, x_{2}\right]\right) \cap B=\emptyset$; let this $\varepsilon_{z}$ be chosen. It remains to show that in every case, $N_{c z} \cap B=\emptyset$. Case 1 is trivial since $N_{c .} \subseteq A$. Assume Case 2, and let $z=\left(z_{1}, z_{2}\right) \in N_{c,} \cap B$. Then $x_{1} \leq z_{1}$ and $x_{2}-\varepsilon<z_{2} \leq x_{2}$. Since $B$ is decreasing, $\left(x_{1}, z_{2}\right) \in B \cap\left\{x_{1}\right\} \times\left(x_{2}-\varepsilon_{2}, x_{2}\right]=\emptyset$, a contradiction. Similar arguments apply in Cases 3 and 4.

Let $y=\left(y_{1}, y_{2}\right) \in B$; we again consider four similar cases in defining $M_{c}(y)$.

CASE a: $\left(-\infty, y_{1}\right]$ open in $X,\left(-\infty, y_{2}\right]$ open in $Y$.

Let $M_{\varepsilon}(x)=\left(-\infty, y_{1}\right] \times\left(-\infty, y_{2}\right]$.

CASE b: $\left(-\infty, y_{1}\right]$ open in $X,\left(-\infty, y_{2}\right]$ not open in $Y$.

Let $M_{c}(y)=\left(-\infty, y_{1}\right] \times\left(-\infty, y_{2}+\varepsilon\right)$.

CASE c: $\left(-\infty, y_{1}\right]$ not open in $X,\left(-\infty, y_{2}\right]$ open in $Y$.

Let $M_{\varepsilon}(y)=\left(-\infty, y_{1}+\varepsilon\right) \times\left(-\infty, y_{2}\right]$.

CASE d: $\left(-\infty, y_{1}\right]$ not open in $X,\left(-\infty, y_{2}\right]$ not open in $Y$.

Let $M_{c}(y)=\left(-\infty, y_{1}+\varepsilon\right) \times\left(-\infty, y_{2}+\varepsilon\right)$. 
CLAIM 2: For each $y \in B$, there is $\varepsilon_{y}>0$ such that $N_{\varepsilon,} \cap A=\emptyset$.

PROOF. (similar to Claim 1)

CLAIM 3: For each $x \in A$ and $y \in B, N_{\frac{s_{2}}{2}}(x) \cap M_{\frac{5 y}{2}}(y)=\emptyset$.

PROOF. There are sixteen cases here to consider, but some are trivial. In particular, Cases 1a, 1b, 1c, 1d, 2a, 3a, and 4a are all trivial since in each of these Cases, either $N_{\frac{4}{3}}(x) \subseteq A$ or $M_{\frac{\sigma_{2}}{2}}(y) \subseteq B$. Here, for example, is another case.

CASE 2b: Suppose $z \in N_{\frac{\sigma_{2}}{2}}(x) \cap M_{\frac{\sigma_{y}}{2}}(y)$. Then $x_{1} \leq z_{1} \leq y_{1}$ and $x_{2}-\frac{\epsilon_{z}}{2}<z_{2}<y_{2}+\frac{c_{y}}{2}$. First, suppose $\varepsilon_{x} \leq \varepsilon_{y}$. Note that $\left(y_{1}, x_{2}\right) \in A$, and $x_{2}-z_{2}<\frac{\varepsilon_{z}}{2}, z_{2}-y_{2}<\frac{c_{y}}{2}$ imply that $x_{2}-y_{2}<\frac{\varepsilon_{z}}{2}+\frac{\varepsilon_{y}}{2} \leq \varepsilon_{y}$. Thus $\left(y_{1}, x_{2}\right) \in A \cap M_{\varepsilon_{y}}(y)$, which is a contradiction. Next, suppose $\varepsilon_{y} \leq \varepsilon_{x}$. Then $\left(x_{1}, y_{2}\right) \in B$, and again $x_{2}-y_{2}<\frac{\varepsilon_{z}}{2}+\frac{\varepsilon_{y}}{2} \varepsilon_{x}$. Therefore, $\left(x_{1}, y_{2}\right) \in B \cap N_{c_{z}}(x)$, again a contradiction. We conclude that $N_{\frac{s_{2}}{2}}(x) \cap M_{\frac{s_{y}}{2}}(y)=\emptyset$.

Case $3 c$ will be very similar to $2 b$. There remain seven cases: $2 c, 2 d, 3 b, 3 d, 4 b, 4 c, 4 d$; the details are repetitious and will be omitted. I

COROLLARY. If $P$ is an ordered plane, then $w_{0} P=\beta_{0} P$ iff $P$ has one of the four topoloties of Theorem 1.

We conclude with an example which shows that a product of subspaces of $R$ with the topologics inherited from $\tau_{u}$ is not necessarily a $c$-space.

EXAMPLE. Let $X$ be the set of rational numbers with the usual topology; let $Y$ be $R$ with the usual topology. Let $a$ be an irrational number; let $b$ be a rational number. Let $\left(a_{n}\right)$ be an increasing sequence of rational numbers converging to $a$ in $Y$. Let $\left(b_{n}\right)$ be a decreasing sequence of real numbers converging to $b$ in $Y$ which is not eventually constant. Then $\left\{\left(a_{n}, b_{n}\right): n \in N\right\}$ is a $c$-set in $X \times Y$. Let $r$ be a rational number greater than $a$; then $(r, b) \notin i(A)$ but $(r, b) \in I(A)$. This shows that $X \times Y$ is not a $c$-space. I

It is natural to ask whether the preceding results generalize to an ordered product $Y=X_{1} \times$ $X_{2} \times \cdots \times X_{n}$, where each $X_{i}$ is a real ordered space. Obviously, $\beta_{0} Y$ and $w_{0} Y$ coincide if $Y$ is discrete. However, it is shown in [4] that $\beta_{0} Y$ and $w_{0} Y$ are not equivalent if $Y$ is equipped with the usual topology of $n$-dimensional Euclidean space for $n \geq 3$. Whether there exist any non-discrete topologies for $Y(n \geq 3)$ such that $\beta_{0} Y=w_{0} Y$ is not presently known.

\section{REFERENCES}

[1] T.H. Choe and Y.S. Park, Wallman's Type Order Compactification, Pacific J. Math. 82 (1979), 339-347.

[2] P. Fletcher and W. Lindgren, Quasi-Uniform Spaces, Lecture Notes in Pure and Applied Mathematics, Vol. 77, Marcel Dekker, Inc., New York (1982).

[3] D.C. Kent, On the Wallman Order Compactification, Pacific J. Math. 118 (1985), 159-163.

[4] D.C. Kent and T.A. Richmond, Separation Properties of the Wallman Ordered Compactification, Internat. J. Math. \& Math. Sci. 13 (1990), 209-222.

[5] L. Nachbin, Topology and Order, Van Nostrand Mathematical Series 4, Princeton, N.J. 1965. 


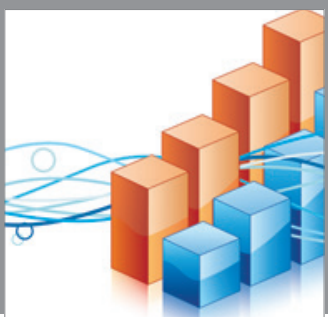

Advances in

Operations Research

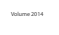

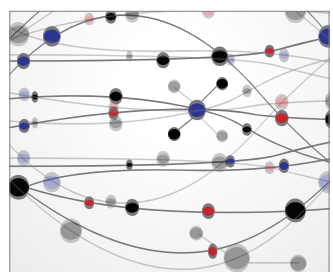

\section{The Scientific} World Journal
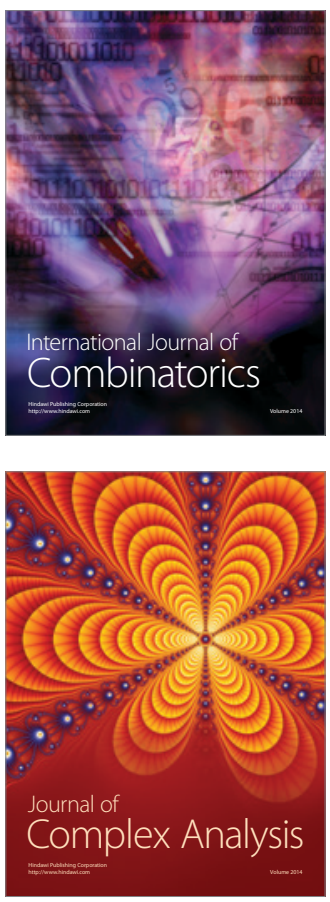

International Journal of

Mathematics and

Mathematical

Sciences
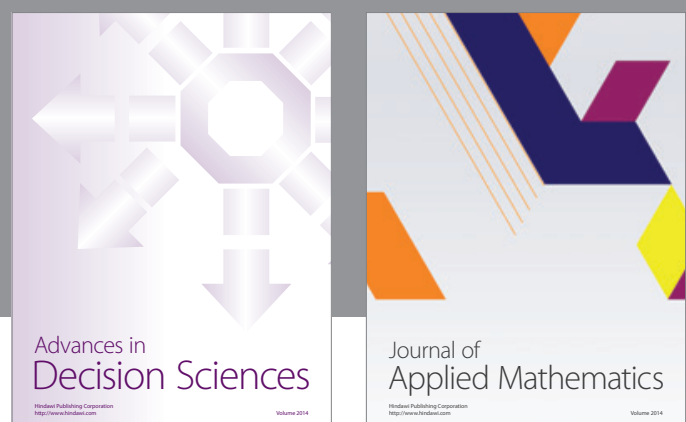

Journal of

Applied Mathematics
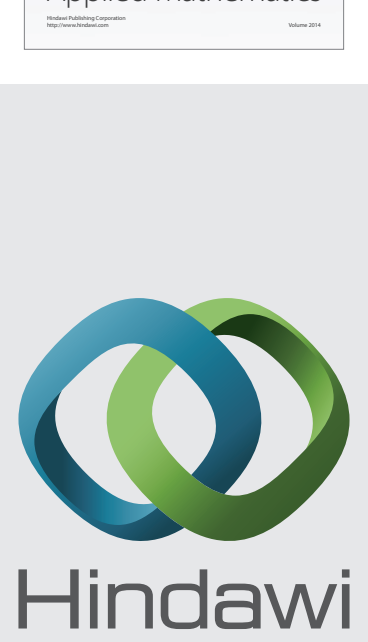

Submit your manuscripts at http://www.hindawi.com
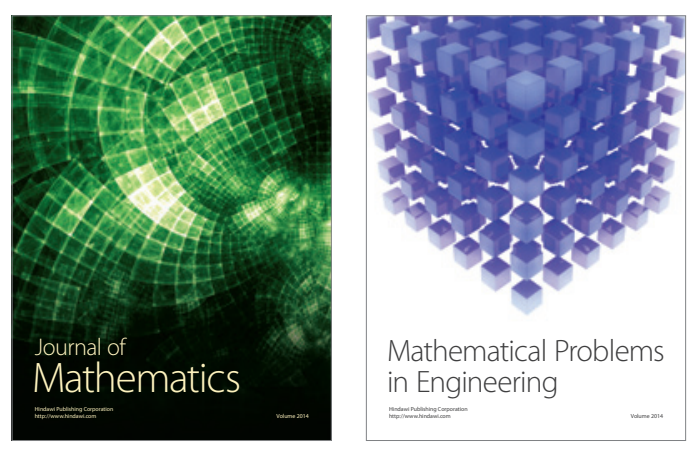

Mathematical Problems in Engineering
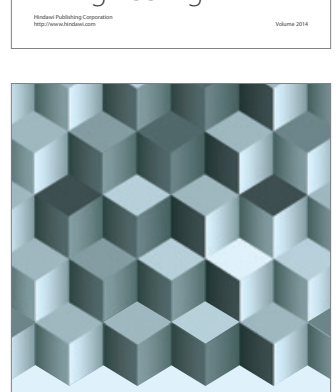

Journal of

Function Spaces
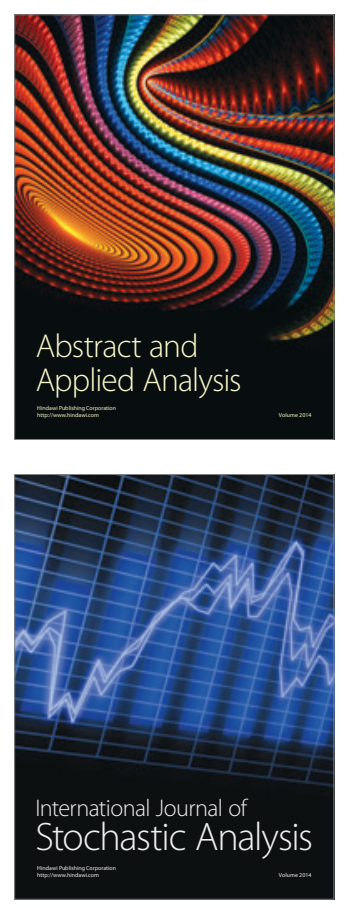

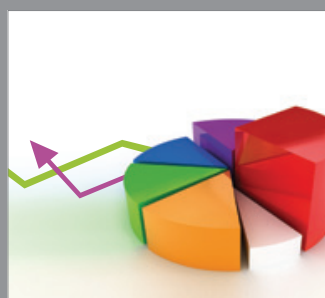

ournal of

Probability and Statistics

Promensencen
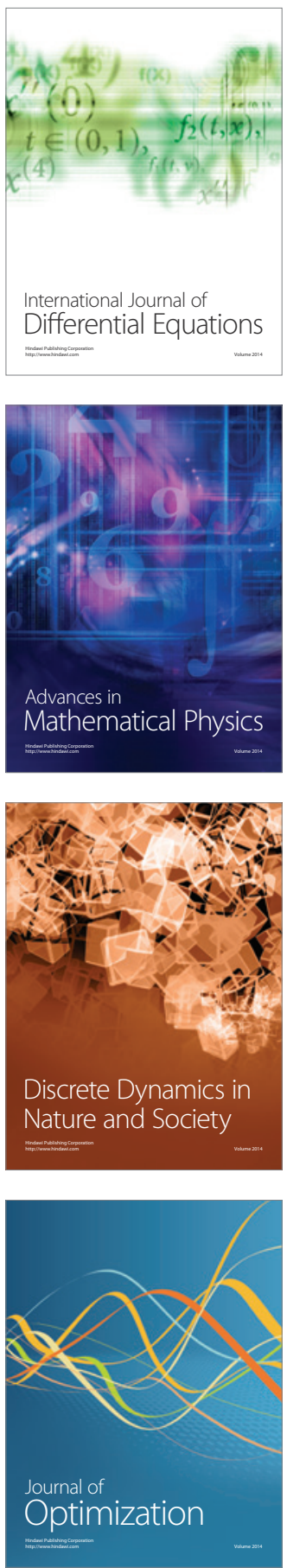